\title{
On the inverse eigenvalue problem of symmetric nonnegative matrices
}

\author{
A. M. Nazari ${ }^{1}$ (1) A. Mashayekhi ${ }^{1}$ A. Nezami ${ }^{1}$
}

Received: 6 February 2018 / Accepted: 30 October 2019 / Published online: 19 December 2019

(c) The Author(s) 2019

\begin{abstract}
In this paper, at first for a given set of real numbers with only one positive number, and in continue for a given set of real numbers in special conditions, we construct a symmetric nonnegative matrix such that the given set is its spectrum.
\end{abstract}

Keywords Symmetric nonnegative inverse eigenvalue problem $\cdot$ Spectrum of matrix $\cdot$ Perron eigenvalue

Mathematics Subject Classification $15 \mathrm{~A} 29 \cdot 15 \mathrm{~A} 18$

\section{Introduction}

The symmetric nonnegative inverse eigenvalue problem (SNIEP) asks for necessary and sufficient conditions on a list $\sigma=\left(\lambda_{1}, \lambda_{2}, \ldots, \lambda_{n}\right)$ of real numbers in order that it be the spectrum of a symmetric nonnegative matrix $A$ with spectrum $\sigma$, we will say that $\sigma$ is symmetrically realizable and that it is symmetric realization of $\sigma$.

Fielder [1] obtained some necessary and some sufficient conditions for a set of $n$ real numbers $\sigma=\left\{\lambda_{1}, \lambda_{2}, \ldots, \lambda_{n}\right\}$ that it to be the set of eigenvalues of $n \times n$ symmetric nonnegative matrix. Sufficient conditions for the SNIEP have been obtained in [2-9]. Soto and Julio [10] showed that most of these sufficient conditions can be obtained by the use of a result by Soto et al. [11]. They tried to find always a solution matrix.

Laffey and Helena Smigoc [12] presented a method that by combination of two symmetric nonnegative matrices with prescribed eigenvalues, find a new symmetric matrix of union of eigenvalues of two matrices except Perron eigenvalues of one of these matrices.

A. M. Nazari

a-nazari@araku.ac.ir

A. Mashayekhi

a-mashayekhi@arashad.araku.ac.ir

A. Nezami

a-nezami@arashad.araku.ac.ir

1 Department of Mathematics, Arak University, P. O.

Box 38156-8-8349, Arak, Iran
One of the special and interesting cases of SNIEP is inverse eigenvalues of Euclidean distance matrix (EDM). For instance, T.L. Hayden, R. Reams and J. Wells have solved the inverse eigenvalue problem for Euclidean distance matrices of order $n=3,4,5,6$, and any $n$ for which there exists a Hadamard matrix and also they solved this problem: If for $n \in \mathbb{N}$ there exists a Hadamard matrix of order $n$, then there is an $(n+1) \times(n+1)$ and an $(n+2) \times(n+2)$ distance matrix with eigenvalues which hold under special conditions for $n \leqslant 16$ [13]. In the paper [14], Jaklič and Modic offered a method for constructing a symmetric nonnegative matrix with zero diagonal and eigenvalues $\lambda_{i}$, where $\sum_{i=1}^{n} \lambda_{i}=0$ and $\lambda_{1}>0>\lambda_{2} \geqslant \cdots \geqslant \lambda_{n}$, then they survey the inverse eigenvalue problem for Euclidean distance matrices, which are a subclass of such matrices. Nazari and Mahdinasab solved this problem without using any Hadamard matrix [15].

Through this paper the following notation is used. The spectral radius of symmetric nonnegative matrix $A$ denoted by $\lambda_{1}=\rho(A)$. There is a right and a left eigenvector associated with the Perron eigenvalue with nonnegative entries. In addition $s_{k}$ the $k$ th power sum of the eigenvalues $\lambda_{i}$ and in the list $\sigma, \lambda_{1}$ is the Perron element.

Some necessary conditions on the list of real number $\sigma=\left(\lambda_{1}, \lambda_{2}, \ldots, \lambda_{n}\right)$ to be the spectrum of a nonnegative matrix are listed below.

(1) The Perron eigenvalue $\max \left\{\left|\lambda_{i}\right| ; \lambda_{i} \in \sigma\right\}$ belongs to $\sigma$ (Perron-Frobenius theorem).

(2) $s_{k}=\sum_{i=1}^{n} \lambda_{i}^{k} \geq 0$. 
(3) $s_{k}^{m} \leq n^{m-1} s_{k m}$ for $k, m=1,2, \ldots$ (JLL inequality) [16, $17]$.

In this paper we use Theorem 2.1 from [18] and again prove the Theorem 2.4 from [1] and find a solution for SNIEP by a recursive method. The Theorem 2.1 is similar to the Lemma 5 of [2].

\section{Construction}

Theorem 2.1 [18] Let $B$ be a $m \times m$ nonnegative matrix, $M_{1}=\left\{\mu_{1}, \mu_{2}, \ldots, \mu_{m}\right\}$ be its eigenvalues and $\mu_{1}$ be Perron eigenvalue of $B$. Also assume that $A$ is an $n \times n$ nonnegative matrix in following form

$$
A=\left(\begin{array}{ll}
A_{1} & a \\
b^{T} & \mu_{1}
\end{array}\right),
$$

where $A_{1}$ is an $(n-1) \times(n-1)$ matrix, $a$ and $b$ are arbitrary vectors in $\mathbb{R}^{n-1}$ and $M_{2}=\left\{\lambda_{1}, \lambda_{2}, \ldots, \lambda_{n}\right\}$ is the set of eigenvalues of $A$. Then there exists an $(m+n-1) \times(m+n-1)$ nonnegative matrix such that $M=\left\{\mu_{2}, \ldots, \mu_{m}, \lambda_{1}, \lambda_{2}, \ldots, \lambda_{n}\right\}$ is its eigenvalues.

In above Theorem, this is very important that the Perron eigenvalue of matrix $B$ exactly lies on the main diagonal of matrix $A$. In the following Theorem (Fiedler [1]) that we again prove by mathematical induction, we try to lie the Perron eigenvalue of matrix $B$ on the main diagonal of matrix $A$. This solution of problem can be constructed by $C=\left(\begin{array}{cc}A_{1} & a s^{*} \\ s b^{T} & B\end{array}\right)$, where $s$ is normalized eigenvector corrsponding to the Perron eigenvalue of $B$ and $s^{*}$ is transpose conjugate of vector $s$ and $b^{T}$ is transpose of vector $b$.

Theorem 2.2 Let $\sigma=\left\{\lambda_{1}, \lambda_{2}, \ldots, \lambda_{n}\right\}$ be a set of real numbers such that only $\lambda_{1}$ is a positive number and

$\lambda_{1}+\lambda_{2}+\cdots+\lambda_{n} \geq 0$,

then there exists an $n \times n$ symmetric nonnegative matrix that $\sigma$ is its spectrum.

Proof We provide proof by induction on $n$. Let $n=2$, then by Fiedler [1] the following symmetric nonnegative $2 \times 2$ matrix is solution

$$
A=\left(\begin{array}{cc}
0 & \sqrt{-\lambda_{1} \lambda_{2}} \\
\sqrt{-\lambda_{1} \lambda_{2}} & \lambda_{1}+\lambda_{2}
\end{array}\right) .
$$

For $n=3$, we set $\sigma_{1}=\left\{\lambda_{1}, \lambda_{2}\right\}$ then the symmetric nonnegative matrix (2.2) realizes $\sigma_{1}$. Now let $\sigma_{2}=\left\{\lambda_{1}+\lambda_{2}, \lambda_{3}\right\}$, then the following matrix realizes $\sigma_{2}$,

$B=\left(\begin{array}{cc}0 & \sqrt{-\left(\lambda_{1}+\lambda_{2}\right) \lambda_{3}} \\ \sqrt{-\left(\lambda_{1}+\lambda_{2}\right) \lambda_{3}} & \lambda_{1}+\lambda_{2}+\lambda_{3}\end{array}\right)$.

Since $\lambda_{1}+\lambda_{2} \geq\left|\lambda_{3}\right|$, then $\lambda_{1}+\lambda_{2}$ is the Perron eigenvalue of $B$. The normalized eigenvector corresponding to $\lambda_{1}+\lambda_{2}$ is

$s=\left(\sqrt{\frac{-\lambda_{3}}{\lambda_{1}+\lambda_{2}-\lambda_{3}}}, \sqrt{\frac{\lambda_{1}+\lambda_{2}}{\lambda_{1}+\lambda_{2}-\lambda_{3}}}\right)^{T}$.

Whereas $\lambda_{1}+\lambda_{2}$ set on the main diagonal of $A$ and $a=\left(\sqrt{-\lambda_{1} \lambda_{2}}\right)$, so

$a s^{T}=\left(\frac{\sqrt{\lambda_{1} \lambda_{2} \lambda_{3}}}{\sqrt{\lambda_{1}+\lambda_{2}-\lambda_{3}}} \frac{\sqrt{-\left(\lambda_{1}+\lambda_{2}\right) \lambda_{1} \lambda_{2}}}{\sqrt{\lambda_{1}+\lambda_{2}-\lambda_{3}}}\right)$,

then by Theorem 2.1 the symmetric nonnegative matrix

$$
\begin{aligned}
A & =\left(\begin{array}{cc}
A_{1} & a s^{T} \\
s a^{T} & B
\end{array}\right) \\
& =\left(\begin{array}{ccc}
0 & \frac{\sqrt{\lambda_{1} \lambda_{2} \lambda_{3}}}{\sqrt{\lambda_{1}+\lambda_{2}-\lambda_{3}}} & \frac{\sqrt{-\left(\lambda_{1}+\lambda_{2}\right) \lambda_{1} \lambda_{2}}}{\sqrt{\lambda_{1}+\lambda_{2}-\lambda_{3}}} \\
\frac{\sqrt{\lambda_{1} \lambda_{2} \lambda_{3}}}{\sqrt{\lambda_{1}+\lambda_{2}-\lambda_{3}}} & 0 & \sqrt{-\left(\lambda_{1}+\lambda_{2}\right) \lambda_{3}} \\
\frac{\sqrt{-\left(\lambda_{1}+\lambda_{2}\right) \lambda_{1} \lambda_{2}}}{\sqrt{\lambda_{1}+\lambda_{2}-\lambda_{3}}} & \sqrt{-\left(\lambda_{1}+\lambda_{2}\right) \lambda_{3}} & \lambda_{1}+\lambda_{2}+\lambda_{3}
\end{array}\right),
\end{aligned}
$$

has the eigenvalues $\sigma=\left\{\lambda_{1}, \lambda_{2}, \lambda_{3}\right\}$.

Now let $\sigma=\left\{\lambda_{1}, \lambda_{2}, \ldots, \lambda_{k}, \lambda_{k+1}\right\}$ be a set of numbers such that $\lambda_{1}$ is the only positive element of $\sigma$ and $\lambda_{1}+\lambda_{2}+\cdots+\lambda_{k+1} \geq 0$. Assume that $\sigma_{1}=\left\{\lambda_{1}, \lambda_{2}, \ldots, \lambda_{k}\right\}$ and $L_{k}=\lambda_{1}+\lambda_{2}+\cdots+\lambda_{k}$, for $k=1,2, \cdots$, then $L_{k} \geq\left|\lambda_{k+1}\right|$ therefore by hypothesis of induction and continuing the above process, we can construct the symmetric nonnegative matrix

$A=\left(\begin{array}{cc}A_{1} & a \\ a^{T} & L_{k}\end{array}\right)$,

with the eigenvalues $\left\{\lambda_{1}, \lambda_{2}, \ldots, \lambda_{k}\right\}$, where $A_{1}$ is a $(k-1) \times(k-1)$ symmetric nonnegative matrix and $a \in \mathbb{R}^{k-1}$ (recall that the $(2,2)$ entry of symmetric matrix (2.2) and the $(3,3)$ entry of symmetric matrix (2.3) are $L_{2}$ and $L_{3}$, respectively). On the other hand the matrix 
$B=\left(\begin{array}{cc}0 & \sqrt{-L_{k} \lambda_{k+1}} \\ \sqrt{-L_{k} \lambda_{k+1}} & L_{k}+\lambda_{k+1}\end{array}\right)$,

has eigenvalues $\left\{L_{k}, \lambda_{k+1}\right\}$. It is clear that $L_{k}$ is the Perron eigenvalue of this matrix and its normalized eigenvector is $s=\left(\sqrt{\frac{-\lambda_{k+1}}{L_{k}-\lambda_{k+1}}}, \sqrt{\frac{L_{k}}{L_{k}-\lambda_{k+1}}}\right)^{T}$. By noting that the matrices $A$ and $B$ satisfy the condition of Theorem 2.1, then by this theorem the following nonnegative matrix

$C=\left(\begin{array}{cc}A_{1} & a s^{T} \\ s a^{T} & B\end{array}\right)$,

has eigenvalues $\sigma_{1}=\left\{\lambda_{1}, \lambda_{2}, \ldots, \lambda_{k}, \lambda_{k+1}\right\}$.

In continue we try to construct a symmetric nonnegative matrix for a given set of real numbers with nonnegative summation that holds in special conditions.

\section{The case $n=2$}

For $n=2$ we have only two disjoint cases that brings in following Theorem.

Theorem 3.1 Let $\sigma=\left\{\lambda_{1}, \lambda_{2}\right\}$ be a set of two real numbers such that $\lambda_{1} \geq\left|\lambda_{2}\right|$. Then $\sigma$ is the set of eigenvalues of a symmetric nonnegative matrix.

Proof $\sigma$ has only one of following cases:

(a) If $\lambda_{2} \geq 0$, then $A=\operatorname{diag}\left(\lambda_{1}, \lambda_{2}\right)$ is a solution of problem.

(b) If $\lambda_{2}<0$, then the matrix

$A=\left(\begin{array}{cc}0 & \sqrt{-\lambda_{1} \lambda_{2}} \\ \sqrt{-\lambda_{1} \lambda_{2}} & \lambda_{1}+\lambda_{2}\end{array}\right)$,

solves the problem.

\section{The case $n=3$}

For $n=3$ if necessary condition $\left(s_{1}=\lambda_{1}+\lambda_{2}+\lambda_{3} \geqslant 0\right)$ is hold, then we can find solution for all cases of $\lambda_{2}$ and $\lambda_{3}$.

Theorem 4.1 Let $\sigma=\left\{\lambda_{1}, \lambda_{2}, \lambda_{3}\right\}$ be a set of real numbers. Then there exists a symmetric nonnegative matrix that realizes $\sigma$, if it satisfies in the following conditions:

$\lambda_{1}+\lambda_{2}+\lambda_{3} \geq 0$ $\lambda_{1} \in \mathbb{R}, \lambda_{1} \geq\left|\lambda_{i}\right| ; i=2,3$.

Proof By the above conditions we have the following cases:

(a) If $\lambda_{2}, \lambda_{3} \geq 0$, then the nonnegative matrix $C=\operatorname{diag}\left(\lambda_{1}, \lambda_{2}, \lambda_{3}\right)$ is a solution of our problem.

(b) If $\lambda_{2}, \lambda_{3}<0$, then by Theorem 2.2 the following symmetric nonnegative matrix is solution of our problem

$A=\left(\begin{array}{ccc}0 & \frac{\sqrt{\lambda_{1} \lambda_{2} \lambda_{3}}}{\sqrt{\lambda_{1}+\lambda_{2}-\lambda_{3}}} & \frac{\sqrt{-\left(\lambda_{1}+\lambda_{2}\right) \lambda_{1} \lambda_{2}}}{\sqrt{\lambda_{1}+\lambda_{2}-\lambda_{3}}} \\ \frac{\sqrt{\lambda_{1} \lambda_{2} \lambda_{3}}}{\sqrt{\lambda_{1}+\lambda_{2}-\lambda_{3}}} & 0 & \sqrt{-\left(\lambda_{1}+\lambda_{2}\right) \lambda_{3}} \\ \frac{\sqrt{-\left(\lambda_{1}+\lambda_{2}\right) \lambda_{1} \lambda_{2}}}{\sqrt{\lambda_{1}+\lambda_{2}-\lambda_{3}}} & \sqrt{-\left(\lambda_{1}+\lambda_{2}\right) \lambda_{3}} & \lambda_{1}+\lambda_{2}+\lambda_{3}\end{array}\right)$.

(c) If $\lambda_{2}<0$ and $\lambda_{3} \geq 0$, then the symmetric nonnegative matrix

$C=\left(\begin{array}{ll}A_{1} & a \\ a^{T} & \lambda_{3}\end{array}\right)$,

is a solution of our problem, where $A_{1}$ is matrix (3.1) and $a$ is zero vector with dimension of $2 \times 1$.

\section{The case $n=4$}

For $n=4$ if holds only the necessary condition $s_{1}=\lambda_{1}+\lambda_{2}+\lambda_{3}+\lambda_{4} \geq 0$ with Perron eigenvalue $\lambda_{1}$, then the SINEP problem has solution. We consider 5 different cases that brings in following Theorem.

Theorem 5.1 Let $\sigma=\left\{\lambda_{1}, \lambda_{2}, \lambda_{3}, \lambda_{4}\right\}$ be a set of real numbers with the following conditions

$\lambda_{1}+\lambda_{2}+\lambda_{3}+\lambda_{4} \geq 0$

$\lambda_{1} \geq\left|\lambda_{i}\right|, \quad i=2,3,4$.

Then there exists a symmetric nonnegative matrix that $\sigma$ is its spectrum.

Proof In accordance with the above conditions we consider the following cases:

(a) If $\lambda_{2}, \lambda_{3}, \lambda_{4} \geq 0$, then the nonnegative matrix $C=\operatorname{diag}\left(\lambda_{1}, \lambda_{2}, \lambda_{3}, \lambda_{4}\right)$ is a desired matrix.

(b) If $\lambda_{2}, \lambda_{3}, \lambda_{4}<0$, then by using Theorem 2.2 , the following symmetric nonnegative matrix has spectrum $\sigma$ : 
$C=\left(\begin{array}{cccc}0 & \frac{\sqrt{\lambda_{1} \lambda_{2} \lambda_{3}}}{\sqrt{\lambda-\lambda_{3}}} & \frac{\sqrt{\lambda_{1} \lambda_{2} \lambda_{4} \lambda}}{\sqrt{\left(\lambda-\lambda_{3}\right)\left(\lambda^{\prime}-\lambda_{4}\right)}} & \frac{\sqrt{-\lambda^{\prime} \lambda \lambda_{1} \lambda_{2}}}{\sqrt{\left(\lambda-\lambda_{3}\right)\left(\lambda^{\prime}-\lambda_{4}\right)}} \\ \frac{\sqrt{\lambda_{1} \lambda_{2} \lambda_{3}}}{\sqrt{\lambda-\lambda_{3}}} & 0 & \frac{\sqrt{\lambda_{3} \lambda_{4} \lambda}}{\sqrt{\left(\lambda^{\prime}-\lambda_{4}\right)}} & \frac{\sqrt{-\lambda_{3} \lambda \lambda^{\prime}}}{\sqrt{\left(\lambda^{\prime}-\lambda_{4}\right)}} \\ \frac{\sqrt{\lambda_{1} \lambda_{2} \lambda_{4} \lambda}}{\sqrt{\left(\lambda-\lambda_{3}\right)\left(\lambda^{\prime}-\lambda_{4}\right)}} & \frac{\sqrt{\lambda_{3} \lambda_{4} \lambda}}{\sqrt{\left(\lambda^{\prime}-\lambda_{4}\right)}} & 0 & \sqrt{-\lambda^{\prime} \lambda_{4}} \\ \frac{\sqrt{-\lambda^{\prime} \lambda \lambda_{1} \lambda_{2}}}{\sqrt{\left(\lambda-\lambda_{3}\right)\left(\lambda^{\prime}-\lambda_{4}\right)}} & \frac{\sqrt{-\lambda_{3} \lambda \lambda^{\prime}}}{\sqrt{\left(\lambda^{\prime}-\lambda_{4}\right)}} & \sqrt{-\lambda^{\prime} \lambda_{4}} & \lambda^{\prime}+\lambda_{4}\end{array}\right)$,

where $\lambda=\lambda_{1}+\lambda_{2}, \lambda^{\prime}=\lambda_{1}+\lambda_{2}+\lambda_{3}$.

(c) If $\lambda_{2}<0$ and $\lambda_{3}, \lambda_{4} \geq 0$, then the symmetric nonnegative matrix

$C=\left(\begin{array}{ll}A_{1} & a \\ a^{T} & \lambda_{4}\end{array}\right)$

is a solution of this problem, where $A_{1}$ is matrix (4.4) and $a$ is zero vector with dimension of $3 \times 1$.

(d) If $\lambda_{2}, \lambda_{3} \leq 0, \lambda_{4}>0$ and at least for one of the eigenvalues $\lambda_{2}$ and $\lambda_{3}$, for example $\lambda_{3}$, we have $\lambda_{3}+\lambda_{4} \geq 0$, then the nonnegative matrix

$C=\left(\begin{array}{cccc}0 & \sqrt{-\lambda_{1} \lambda_{2}} & 0 & 0 \\ \sqrt{-\lambda_{1} \lambda_{2}} & \lambda_{1}+\lambda_{2} & 0 & 0 \\ 0 & 0 & 0 & \sqrt{-\lambda_{3} \lambda_{4}} \\ 0 & 0 & \sqrt{-\lambda_{3} \lambda_{4}} & \lambda_{3}+\lambda_{4}\end{array}\right)$,

is a solution of our problem.

(e) If $\lambda_{2}, \lambda_{3} \leq 0, \lambda_{4}>0$ and we have $\lambda_{2}+\lambda_{4} \leq 0, \lambda_{3}+\lambda_{4} \leq 0$, then by Lowey and London [16] we consider $C=\left(c_{i j}\right)$ in the following form

$C=U\left(\begin{array}{cccc}\lambda_{2} & & & \\ & \lambda_{3} & & \\ & & \lambda_{4} & \\ & & & \lambda_{1}\end{array}\right) U^{T}$,

where $U$ is orthogonal matrix

$U=\frac{1}{2}\left(\begin{array}{cccc}1 & 1 & 1 & 1 \\ -1 & -1 & 1 & 1 \\ 1 & -1 & -1 & 1 \\ -1 & 1 & -1 & 1\end{array}\right)$

We have also the following relations:

$c_{11}=c_{22}=c_{33}=c_{44}=\frac{1}{4}\left(\lambda_{1}+\lambda_{2}+\lambda_{3}+\lambda_{4}\right) \geq 0$

$c_{12}=c_{21}=c_{34}=c_{43}=\frac{1}{4}\left(\lambda_{1}-\left(\lambda_{2}+\lambda_{3}\right)+\lambda_{4}\right) \geq 0$

$c_{13}=c_{31}=c_{24}=c_{42}=\frac{1}{4}\left(\left(\lambda_{1}+\lambda_{2}\right)-\left(\lambda_{3}+\lambda_{4}\right)\right) \geq 0$

$c_{14}=c_{41}=c_{23}=c_{32}=\frac{1}{4}\left(\left(\lambda_{1}+\lambda_{3}\right)-\left(\lambda_{2}+\lambda_{4}\right)\right) \geq 0$.
So $C$ is a symmetric nonnegative matrix and solves our problem.

\section{The case $n=5$}

Theorem 6.1 Let $\sigma=\left\{\lambda_{1}, \lambda_{2}, \lambda_{3}, \lambda_{4}, \lambda_{5}\right\}$ be a set of real numbers and satisfies in the following conditions

$\lambda_{1}+\lambda_{2}+\lambda_{3}+\lambda_{4}+\lambda_{5} \geq 0$

$\lambda_{1} \in \mathbb{R}, \lambda_{1} \geq\left|\lambda_{i}\right| ; i=2,3,4,5$,

if $\left(\lambda_{2}, \lambda_{3}<0, \lambda_{4}, \lambda_{5} \geq 0\right) \longrightarrow \lambda_{1}+\lambda_{2}+\lambda_{3} \geq 0$,

if $\left(\lambda_{2}, \lambda_{3}, \lambda_{4}<0, \lambda_{5} \geq 0\right) \longrightarrow \lambda_{1}+\lambda_{2}+\lambda_{3}+\lambda_{4} \geq 0$.

Then there exists a symmetric nonnegative matrix that realize $\sigma$.

Proof We present the proof in following cases.

(a) If $\lambda_{2}, \lambda_{3}, \lambda_{4}, \lambda_{5} \geq 0$, then the symmetric nonnegative matrix $C=\operatorname{diag}\left(\lambda_{1}, \lambda_{2}, \lambda_{3}, \lambda_{4}, \lambda_{5}\right)$ is solution.

(b) If $\lambda_{2}, \lambda_{3}, \lambda_{4}, \lambda_{5}<0$, then by Theorem 2.2, we construct the solution. Since the symmetric nonnegative matrix(5.3), with the spectrum $\sigma_{1}=\left\{\lambda_{1}, \lambda_{2}, \lambda_{3}, \lambda_{4}\right\}$ and nonnegative matrix

$B=\left(\begin{array}{cc}0 & \sqrt{-\left(\lambda_{1}+\lambda_{2}+\lambda_{3}+\lambda_{4}\right) \lambda_{5}} \\ \sqrt{-\left(\lambda_{1}+\lambda_{2}+\lambda_{3}+\lambda_{4}\right) \lambda_{5}} & \lambda_{1}+\lambda_{2}+\lambda_{3}+\lambda_{4}+\lambda_{5}\end{array}\right)$,

with the spectrum $\sigma_{2}=\left\{\lambda_{1}+\lambda_{2}+\lambda_{3}+\lambda_{4}, \lambda_{5}\right\}$ have the conditions of matrices $A$ and $B$ of Theorem 2.1, respectively, the normalized eigenvector corresponding to the Perron eigenvalue of $\lambda=\lambda_{1}+\lambda_{2}+\lambda_{3}+\lambda_{4}$ of nonnegative matrix $B$ has the form

$s=\left(\sqrt{\frac{-\lambda_{5}}{\lambda_{1}+\lambda_{2}+\lambda_{3}+\lambda_{4}-\lambda_{5}}}, \sqrt{\frac{\lambda_{1}+\lambda_{2}+\lambda_{3}+\lambda_{4}}{\lambda_{1}+\lambda_{2}+\lambda_{3}+\lambda_{4}-\lambda_{5}}}\right)^{T}$,

then by Theorem 2.2 the following matrix is solution

$C=\left(\begin{array}{ccccc}0 & \frac{\sqrt{\lambda_{1} \lambda_{2} \lambda_{3}}}{\sqrt{\lambda-\lambda_{3}}} & \frac{\sqrt{\lambda_{1} \lambda_{2} \lambda_{4} \lambda}}{\sqrt{\left(\lambda-\lambda_{3}\right)\left(\lambda^{\prime}-\lambda_{4}\right)}} & \frac{\sqrt{\lambda^{\prime} \lambda \lambda_{1} \lambda_{2} \lambda_{5}}}{z} & \frac{\sqrt{-\lambda \lambda^{\prime} \lambda^{\prime \prime} \lambda_{1} \lambda_{2}}}{z} \\ \frac{\sqrt{\lambda_{1} \lambda_{2} \lambda_{3}}}{\sqrt{\lambda-\lambda_{3}}} & 0 & \frac{\sqrt{\lambda_{3} \lambda_{4} \lambda}}{\sqrt{\left(\lambda^{\prime}-\lambda_{4}\right)}} & \frac{\sqrt{\lambda_{3} \lambda_{5} \lambda \lambda^{\prime}}}{y} & \frac{\sqrt{-\lambda_{3} \lambda \lambda^{\prime} \lambda^{\prime \prime}}}{y} \\ \frac{\sqrt{\lambda_{1} \lambda_{2} \lambda_{4} \lambda}}{\sqrt{\left(\lambda-\lambda_{3}\right)\left(\lambda^{\prime}-\lambda_{4}\right)}} & \frac{\sqrt{\lambda_{3} \lambda_{4} \lambda}}{\sqrt{\left(\lambda^{\prime}-\lambda_{4}\right)}} & 0 & \frac{\sqrt{\lambda_{4} \lambda_{5} \lambda^{\prime}}}{\sqrt{\lambda^{\prime \prime}-\lambda_{5}}} & \frac{\sqrt{-\lambda_{4} \lambda^{\prime} \lambda^{\prime \prime}}}{\sqrt{\lambda^{\prime \prime}-\lambda_{5}}} \\ \frac{\sqrt{\lambda^{\prime} \lambda \lambda_{1} \lambda_{2} \lambda_{5}}}{z} & \frac{\sqrt{\lambda_{3} \lambda_{5} \lambda \lambda^{\prime}}}{y} & \frac{\sqrt{\lambda_{4} \lambda_{5} \lambda^{\prime}}}{\sqrt{\lambda^{\prime \prime}-\lambda_{5}}} & 0 & \sqrt{-\lambda^{\prime \prime} \lambda_{5}} \\ \frac{\sqrt{-\lambda \lambda^{\prime} \lambda^{\prime \prime} \lambda_{1} \lambda_{2}}}{z} & \frac{\sqrt{-\lambda_{3} \lambda \lambda^{\prime} \lambda^{\prime \prime}}}{y} & \frac{\sqrt{-\lambda_{4} \lambda^{\prime} \lambda^{\prime \prime}}}{\sqrt{\lambda^{\prime \prime}-\lambda_{5}}} & \sqrt{-\lambda^{\prime \prime} \lambda_{5}} & \lambda^{\prime \prime}+\lambda_{5}\end{array}\right)$, 
where $\lambda=\lambda_{1}+\lambda_{2}, \lambda^{\prime}=\lambda_{1}+\lambda_{2}+\lambda_{3}, \lambda^{\prime \prime}=\lambda_{1}+\lambda_{2}+\lambda_{3}+\lambda_{4}$, $z=\sqrt{\left(\lambda-\lambda_{3}\right)\left(\lambda^{\prime}-\lambda_{4}\right)\left(\lambda^{\prime \prime}-\lambda_{5}\right)}, y=\sqrt{\left(\lambda^{\prime}-\lambda_{4}\right)\left(\lambda^{\prime \prime}-\lambda_{5}\right)}$.

(c) If $\lambda_{2}<0$ and $\lambda_{3}, \lambda_{4}, \lambda_{5} \geq 0$, then the nonnegative matrix

$C=\left(\begin{array}{cc}A & a \\ a^{T} & \lambda_{5}\end{array}\right)$

is a solution of our problem, where $A$ is matrix (5.4) and $a$ is zero vectors with dimension of $4 \times 1$.

(d) If $\lambda_{2}, \lambda_{3}<0$ and $\lambda_{4}, \lambda_{5} \geq 0$, then by (6.3) we have $\lambda_{3}+\lambda_{4}+\lambda_{5} \geq 0$, thus the nonnegative matrix

$A=\left(\begin{array}{ccccc}0 & \frac{\sqrt{\lambda_{1} \lambda_{2} \lambda_{3}}}{\sqrt{\lambda_{1}+\lambda_{2}-\lambda_{3}}} & \frac{\sqrt{-\left(\lambda_{1}+\lambda_{2}\right) \lambda_{1} \lambda_{2}}}{\sqrt{\lambda_{1}+\lambda_{2}-\lambda_{3}}} & 0 & 0 \\ \frac{\sqrt{\lambda_{1} \lambda_{2} \lambda_{3}}}{\sqrt{\lambda_{1}+\lambda_{2}-\lambda_{3}}} & 0 & \sqrt{-\left(\lambda_{1}+\lambda_{2}\right) \lambda_{3}} & 0 & 0 \\ \frac{\sqrt{-\left(\lambda_{1}+\lambda_{2}\right) \lambda_{1} \lambda_{2}}}{\sqrt{\lambda_{1}+\lambda_{2}-\lambda_{3}}} & \sqrt{-\left(\lambda_{1}+\lambda_{2}\right) \lambda_{3}} & \lambda_{1}+\lambda_{2}+\lambda_{3} & 0 & 0 \\ 0 & 0 & 0 & \lambda_{4} & 0 \\ 0 & 0 & 0 & 0 & \lambda_{5}\end{array}\right)$,

is a solution of problem.

(e) If $\lambda_{2}, \lambda_{3} \leq 0, \lambda_{4}, \lambda_{5}>0$ and at least for one of the eigenvalues $\lambda_{2}$ and $\lambda_{3}$, for example $\lambda_{3}$, we have $\lambda_{3}+\lambda_{4} \geq 0$, then the nonnegative matrix

$C=\left(\begin{array}{ccccc}0 & \sqrt{-\lambda_{1} \lambda_{2}} & 0 & 0 & 0 \\ \sqrt{-\lambda_{1} \lambda_{2}} & \lambda_{1}+\lambda_{2} & 0 & 0 & 0 \\ 0 & 0 & 0 & \sqrt{-\lambda_{3} \lambda_{4}} & 0 \\ 0 & 0 & \sqrt{-\lambda_{3} \lambda_{4}} & \lambda_{3}+\lambda_{4} & 0 \\ 0 & 0 & 0 & 0 & \lambda_{5}\end{array}\right)$,

is a solution of problem.

(f) If $\lambda_{2}, \lambda_{3}, \lambda_{4}<0$ and $\lambda_{5} \geq 0$, then by (6.4) we have $\lambda_{1}+\lambda_{2}+\lambda_{3}+\lambda_{4} \geq 0$, then the nonnegative matrix

$C=\left(\begin{array}{cc}A & a \\ a^{T} & \lambda_{5}\end{array}\right)$

is a solution of this problem, where $A$ is matrix (5.3) and $a$ is zero vector with dimension of $4 \times 1$.

\section{The case $n=6$}

Theorem 7.1 Let $\sigma=\left\{\lambda_{1}, \lambda_{2}, \lambda_{3}, \lambda_{4}, \lambda_{5}, \lambda_{6}\right\}$ be a set of real numbers and satisfies in the following conditions,

$\lambda_{1}+\lambda_{2}+\lambda_{3}+\lambda_{4}+\lambda_{5}+\lambda_{6} \geq 0$

$\lambda_{1} \in \mathbb{R}, \lambda_{1} \geq\left|\lambda_{i}\right| ; i=2,3,4,5,6$, if $\left(\lambda_{2}, \lambda_{3}<0, \lambda_{4}, \lambda_{5}, \lambda_{6} \geq 0\right) \longrightarrow \lambda_{1}+\lambda_{2}+\lambda_{3} \geq 0$,

if $\left(\lambda_{2}, \lambda_{3}, \lambda_{4}<0, \lambda_{5}, \lambda_{6} \geq 0\right) \longrightarrow \lambda_{1}+\lambda_{2}+\lambda_{3}+\lambda_{4} \geq 0$.

if $\left(\lambda_{2}, \lambda_{3}, \lambda_{4}, \lambda_{5}<0, \lambda_{6} \geq 0\right) \longrightarrow \lambda_{1}+\lambda_{2}+\lambda_{3}+\lambda_{4}+\lambda_{5} \geq 0$.

Then there exists a symmetric nonnegative matrix that realizes $\sigma$.

Proof We present the proof in following cases.

(a) If $\lambda_{2}, \lambda_{3}, \lambda_{4}, \lambda_{5}, \lambda_{6} \geq 0$, then the symmetric nonnegative matrix $C=\operatorname{diag}\left(\lambda_{1}, \lambda_{2}, \lambda_{3}, \lambda_{4}, \lambda_{5}, \lambda_{6}\right)$ is solution.

(b) If $\lambda_{2}, \lambda_{3}, \lambda_{4}, \lambda_{5}, \lambda_{6}<0$, then by Theorem 2.2 , we construct the solution since the symmetric nonnegative matrix(6.5), with the spectrum $\sigma_{1}=\left\{\lambda_{1}, \lambda_{2}, \lambda_{3}, \lambda_{4}, \lambda_{5}\right\}$ and nonnegative matrix

$B=\left(\begin{array}{cc}0 & \sqrt{-\left(\lambda_{1}+\lambda_{2}+\lambda_{3}+\lambda_{4}+\lambda_{5}\right) \lambda_{6}} \\ \sqrt{-\left(\lambda_{1}+\lambda_{2}+\lambda_{3}+\lambda_{4}+\lambda_{5}\right) \lambda_{6}} & \lambda_{1}+\lambda_{2}+\lambda_{3}+\lambda_{4}+\lambda_{5}+\lambda_{6}\end{array}\right)$,

with the spectrum $\sigma_{2}=\left\{\lambda_{1}+\lambda_{2}+\lambda_{3}+\lambda_{4}+\lambda_{5}, \lambda_{6}\right\}$ have the conditions of matrices $A$ and $B$ of theorem 2.1, respectively. The normalized eigenvector corresponding to Perron eigenvalue of $\lambda=\lambda_{1}+\lambda_{2}+\lambda_{3}+\lambda_{4}+\lambda_{5}$ of nonnegative matrix $B$ has the form of

$s=\left(\sqrt{\frac{-\lambda_{6}}{\lambda_{1}+\lambda_{2}+\lambda_{3}+\lambda_{4}+\lambda_{5}-\lambda_{6}}}, \sqrt{\frac{\lambda_{1}+\lambda_{2}+\lambda_{3}+\lambda_{4}+\lambda_{5}}{\lambda_{1}+\lambda_{2}+\lambda_{3}+\lambda_{4}+\lambda_{5}-\lambda_{6}}}\right)^{T}$,

then by Theorem 2.2 the following matrix is solution

$C=\left(\begin{array}{ll}C_{1} & C_{2}\end{array}\right)$,

where

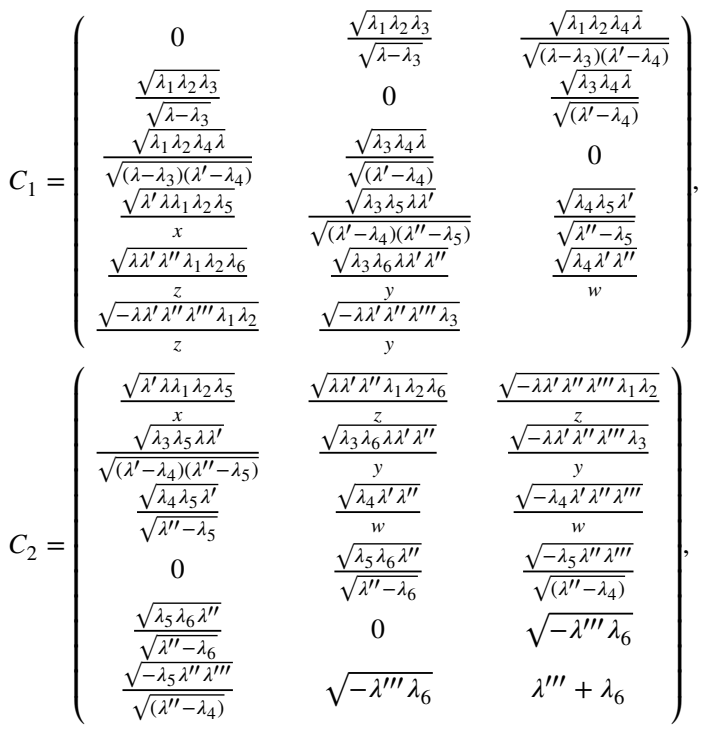


and

$$
\begin{aligned}
\lambda & =\lambda_{1}+\lambda_{2}, \lambda^{\prime}=\lambda_{1}+\lambda_{2}+\lambda_{3}, \\
\lambda^{\prime \prime} & =\lambda_{1}+\lambda_{2}+\lambda_{3}+\lambda_{4}, \lambda^{\prime \prime \prime}=\lambda_{1}+\lambda_{2}+\lambda_{3}+\lambda_{4}+\lambda_{5}, \\
x & =\sqrt{\left(\lambda-\lambda_{3}\right)\left(\lambda^{\prime}-\lambda_{4}\right)\left(\lambda^{\prime \prime}-\lambda_{5}\right)}, \\
z & =\sqrt{\left(\lambda-\lambda_{3}\right)\left(\lambda^{\prime}-\lambda_{4}\right)\left(\lambda^{\prime \prime}-\lambda_{5}\right)\left(\lambda^{\prime \prime \prime}-\lambda_{6}\right)}, \\
y & =\sqrt{\left(\lambda^{\prime}-\lambda_{4}\right)\left(\lambda^{\prime \prime}-\lambda_{5}\right)\left(\lambda^{\prime \prime \prime}-\lambda_{6}\right)}, \\
w & =\sqrt{\left(\lambda^{\prime \prime}-\lambda_{5}\right)\left(\lambda^{\prime \prime \prime}-\lambda_{6}\right)} .
\end{aligned}
$$

(c) If $\lambda_{2}<0$ and $\lambda_{3}, \lambda_{4}, \lambda_{5}, \lambda_{6} \geq 0$, then the nonnegative matrix

$C=\left(\begin{array}{cc}A & a \\ a^{T} & \lambda_{6}\end{array}\right)$

is a solution of this problem, where $A$ is matrix (6.6) and $a, b$ are zero vectors with dimension of $5 \times 1$.

(d) If $\lambda_{2}, \lambda_{3}<0$ and $\lambda_{4}, \lambda_{5}, \lambda_{6} \geq 0$, then by (7.3) we have $\lambda_{1}+\lambda_{2}+\lambda_{3} \geq 0$ then the nonnegative matrix
$C=\left(\begin{array}{cc}A & a \\ a^{T} & \lambda_{6}\end{array}\right)$

is a solution of this problem, where $A$ is matrix (6.9) and $a, b$ are zero vectors with dimension of $5 \times 1$.

(g) If $\lambda_{2}, \lambda_{3}, \lambda_{4}, \lambda_{5}<0$ and $\lambda_{6} \leq 0$, then by (7.5) we have $\lambda_{1}+\lambda_{2}+\lambda_{3}+\lambda_{4}+\lambda_{5} \geq 0$, so the nonnegative matrix

$C=\left(\begin{array}{cc}A & a \\ a^{T} & \lambda_{6}\end{array}\right)$

is a solution of our problem, where $A$ is the matrix (6.5) and $a, b$ are zero vector of dimension $5 \times 1$.

\section{Numerical example}

Example 8.1 Assume given

$\sigma=\left\{\lambda_{1}=7, \lambda_{2}=-4, \lambda_{3}=-4, \lambda_{4}=5, \lambda_{5}=a \geq 0\right\}$,

then we have $\lambda_{3}+\lambda_{4} \geq 0$, consequently by case $(e)$ of Theorem 6.1 the following matrix realized $\sigma$

$$
A=\left(\begin{array}{cccccc}
0 & \frac{\sqrt{\lambda_{1} \lambda_{2} \lambda_{3}}}{\sqrt{\lambda_{1}+\lambda_{2}-\lambda_{3}}} & \frac{\sqrt{-\left(\lambda_{1}+\lambda_{2}\right) \lambda_{1} \lambda_{2}}}{\sqrt{\lambda_{1}+\lambda_{2}-\lambda_{3}}} & 0 & 0 & 0 \\
\frac{\sqrt{\lambda_{1} \lambda_{2} \lambda_{3}}}{\sqrt{\lambda_{1}+\lambda_{2}-\lambda_{3}}} & 0 & \sqrt{-\left(\lambda_{1}+\lambda_{2}\right) \lambda_{3}} & 0 & 0 & 0 \\
\frac{\sqrt{-\left(\lambda_{1}+\lambda_{2}\right) \lambda_{1} \lambda_{2}}}{\sqrt{\lambda_{1}+\lambda_{2}-\lambda_{3}}} & \sqrt{-\left(\lambda_{1}+\lambda_{2}\right) \lambda_{3}} & \lambda_{1}+\lambda_{2}+\lambda_{3} & 0 & 0 & 0 \\
0 & 0 & 0 & \lambda_{4} & 0 & 0 \\
0 & 0 & 0 & 0 & \lambda_{5} & 0 \\
0 & 0 & 0 & 0 & 0 & \lambda_{6}
\end{array}\right) \text {, }
$$

is a solution of problem.

(e) If $\lambda_{2}, \lambda_{3} \leq 0, \lambda_{4}, \lambda_{5}, \lambda_{6}>0$ and at least for one of the eigenvalues $\lambda_{2}$ and $\lambda_{3}$, for example $\lambda_{3}$, we have $\lambda_{3}+\lambda_{4} \geq 0$, then the nonnegative matrix

$C=\left(\begin{array}{cccccc}0 & \sqrt{-\lambda_{1} \lambda_{2}} & 0 & 0 & 0 & 0 \\ \sqrt{-\lambda_{1} \lambda_{2}} & \lambda_{1}+\lambda_{2} & 0 & 0 & 0 & 0 \\ 0 & 0 & 0 & \sqrt{-\lambda_{3} \lambda_{4}} & 0 & 0 \\ 0 & 0 & \sqrt{-\lambda_{3} \lambda_{4}} & \lambda_{3}+\lambda_{4} & 0 & 0 \\ 0 & 0 & 0 & 0 & \lambda_{5} & 0 \\ 0 & 0 & 0 & 0 & 0 & \lambda_{6}\end{array}\right)$,

is a solution of problem.

(f) If $\lambda_{2}, \lambda_{3}, \lambda_{4}<0$ and $\lambda_{5}, \lambda_{6} \geq 0$, then by (7.4) we have $\lambda_{1}+\lambda_{2}+\lambda_{3}+\lambda_{4} \geq 0$, then the nonnegative matrix

$$
C=\left(\begin{array}{ccccc}
0 & \sqrt{28} & 0 & 0 & 0 \\
\sqrt{28} & 3 & 0 & 0 & 0 \\
0 & 0 & 0 & \sqrt{20} & 0 \\
0 & 0 & \sqrt{20} & 1 & 0 \\
0 & 0 & 0 & 0 & a
\end{array}\right) .
$$

Example 8.2 Assume given

$\sigma=\left\{\lambda_{1}=18, \lambda_{2}=-1, \lambda_{3}=-6, \lambda_{4}=-4, \lambda_{5}=-3, \lambda_{6}=-3\right\}$, since $\lambda_{2}, \lambda_{3}, \lambda_{4}, \lambda_{5}, \lambda_{6} \leq 0$ and $\Sigma_{i=1}^{6} \lambda_{i} \geq 0$ then by Theorem 2.2 we construct a solution for $\sigma$. At first it is easy to see that the symmetric matrix $A_{1}=\left[\begin{array}{cc}0 & 3 \sqrt{2} \\ 3 \sqrt{2} & 17\end{array}\right]$ has eigen- 
values $\left[\begin{array}{c}18 \\ -1\end{array}\right]$ and the symmetric matrix $B=\left[\begin{array}{cc}0 & \sqrt{102} \\ \sqrt{102} & 11\end{array}\right]$ has eigenvalues $\left[\begin{array}{c}17 \\ -6\end{array}\right]$, with Perron eigenvector $\left[\begin{array}{l}1 / 23 \sqrt{138} \\ 1 / 23 \sqrt{391}\end{array}\right]$, let $a=[3 \sqrt{2}]$, then the $3 \times 3$ symmetric matrix

$C_{1}=\left(\begin{array}{cc}A_{2} & a s^{*} \\ s a^{T} & B\end{array}\right)=\left[\begin{array}{ccc}0 & \frac{6}{23} \sqrt{69} & \frac{3}{23} \sqrt{2} \sqrt{391} \\ \frac{6}{23} \sqrt{69} & 0 & \sqrt{102} \\ \frac{3}{23} \sqrt{2} \sqrt{391} & \sqrt{102} & 11\end{array}\right]$ has eigenvalues $\left[\begin{array}{c}18 \\ -1 \\ -6\end{array}\right]$, and in next step $B=\left[\begin{array}{cc}0 & 2 \sqrt{11} \\ 2 \sqrt{11} & 7\end{array}\right]$ with eigenvalues $\left[\begin{array}{c}11 \\ -4\end{array}\right]$ and $a=\left[\begin{array}{c}\frac{3}{23} \sqrt{2} \sqrt{391} \\ \sqrt{102}\end{array}\right]$ and Perron eigenvector $s=\left[\begin{array}{c}2 / 15 \sqrt{15} \\ 1 / 15 \sqrt{165}\end{array}\right]$ then the $4 \times 4$ symmetric matrix positive number with zero summation, whose its spectrum is the same as the spectrum of a distance matrix(EDM).

Example 8.3 Assume given

$\sigma=\left\{\lambda_{1}=15, \lambda_{2}=-1, \lambda_{3}=-2, \lambda_{4}=-3, \lambda_{5}=-4, \lambda_{6}=-5\right\}$, since $\lambda_{2}, \lambda_{3}, \lambda_{4}, \lambda_{5}, \lambda_{6} \leq 0$ and $\Sigma_{i=1}^{6} \lambda_{i} \geq 0$ then by Theorem 2.2 we construct a solution for $\sigma$. At first it is easy to see that the symmetric matrix $A_{1}=\left[\begin{array}{cc}0 & \sqrt{15} \\ \sqrt{15} & 14\end{array}\right]$ has eigenvalues $\left[\begin{array}{c}15 \\ -1\end{array}\right]$ and the symmetric matrix $B=\left[\begin{array}{cc}0 & 2 \sqrt{7} \\ 2 \sqrt{7} & 12\end{array}\right]$ has eigenvalues $\left[\begin{array}{c}14 \\ -2\end{array}\right]$, with Perron eigenvector $\left[\begin{array}{cc}\frac{1}{16} & \sqrt{32} \\ \frac{1}{16} & \sqrt{224}\end{array}\right]$, then let $a=[2 \sqrt{7}]$, then the $3 \times 3$ symmetric matrix

$C_{1}=\left(\begin{array}{cc}A_{2} & a s^{*} \\ s a^{T} & B\end{array}\right)=\left[\begin{array}{ccc}0 & \frac{1}{2} \sqrt{2} \sqrt{14} & \frac{8}{16} \sqrt{7} \sqrt{14} \\ \frac{1}{2} \sqrt{2} \sqrt{14} & 0 & 2 \sqrt{7} \\ \frac{8}{16} \sqrt{7} \sqrt{14} & 2 \sqrt{7} & 12\end{array}\right]$ has eigenvalues $\left[\begin{array}{c}15 \\ -1 \\ -2\end{array}\right]$. With continue this method and round

$C=\left(\begin{array}{cc}A_{2} & a s^{*} \\ s a^{T} & B\end{array}\right)=\left[\begin{array}{cccc}0 & \frac{3}{23} \sqrt{2} \sqrt{138} & \frac{2}{115} \sqrt{2} \sqrt{391} \sqrt{15} & \frac{1}{115} \sqrt{2} \sqrt{391} \sqrt{165} \\ \frac{3}{23} \sqrt{2} \sqrt{138} & 0 & 2 / 15 \sqrt{102} \sqrt{15} & 1 / 15 \sqrt{102} \sqrt{165} \\ \frac{2}{115} \sqrt{2} \sqrt{391} \sqrt{15} & 2 / 15 \sqrt{102} \sqrt{15} & 0 & 2 \sqrt{11} \\ \frac{1}{115} \sqrt{2} \sqrt{391} \sqrt{165} & 1 / 15 \sqrt{102} \sqrt{165} & 2 \sqrt{11} & 7\end{array}\right]$

has eigenvalues $\left[\begin{array}{c}18 \\ -6 \\ -4 \\ -1\end{array}\right]$. With continue this method in 3 cases and round the solution with 5 floating point, we have the following solution for $\sigma$ :

$\left[\begin{array}{cccccc}0.0 & 2.1668 & 1.8834 & 1.7108 & 1.7108 & 1.9755 \\ 2.1668 & 0.0 & 5.2155 & 4.7371 & 4.7371 & 5.4702 \\ 1.8834 & 5.2155 & 0.0 & 3.6332 & 3.6331 & 4.1953 \\ 1.7108 & 4.7371 & 3.6332 & 0.0 & 3.0 & 3.4642 \\ 1.7108 & 4.7371 & 3.6331 & 3.0 & 0.0 & 3.4642 \\ 1.9755 & 5.4702 & 4.1953 & 3.4642 & 3.4642 & 1.0\end{array}\right]$.

In the next example, we try to find a symmetric nonnegative matrix for a given set of real numbers that only has a the solution with 5 floating point, we have

$\left[\begin{array}{cccccc}0.0 & 1.36931 & 1.62019 & 1.79743 & 1.90647 & 1.90647 \\ 1.36931 & 0.0 & 2.36643 & 2.62532 & 2.78457 & 2.78457 \\ 1.62019 & 2.36643 & 0.0 & 3.32820 & 3.53009 & 3.53009 \\ 1.79743 & 2.62532 & 3.32820 & 0.0 & 4.24264 & 4.24264 \\ 1.90647 & 2.78457 & 3.53009 & 4.24264 & 0.0 & 5 \\ 1.90647 & 2.78457 & 3.53009 & 4.24264 & 5 & 0.0\end{array}\right]$.

But in Example 2.10. [15], if we choose

$$
W^{\prime}=\left[\begin{array}{ccccc}
1 & -2 & 3 & 4 & -5 \\
-1 & -2 & 3 & -4 & 5 \\
1 & 2 & 3 & -4 & 5 \\
-1 & 2 & -3 & 4 & 5 \\
1 & -2 & -3 & 4 & -5 \\
-1 & 2 & -3 & -4 & -5
\end{array}\right]
$$


then

$D=\left[\begin{array}{cccccc}0 & \frac{49}{15} & \frac{18}{5} & \frac{353}{120} & \frac{159}{40} & \frac{77}{30} \\ \frac{49}{15} & 0 & \frac{5}{3} & \frac{121}{40} & \frac{55}{24} & \frac{17}{5} \\ \frac{18}{5} & \frac{5}{3} & 0 & \frac{323}{120} & \frac{21}{8} & \frac{46}{15} \\ \frac{353}{120} & \frac{121}{40} & \frac{323}{120} & 0 & \frac{77}{30} & \frac{35}{8} \\ \frac{159}{40} & \frac{55}{24} & \frac{21}{8} & \frac{77}{30} & 0 & \frac{353}{120} \\ \frac{77}{30} & \frac{17}{5} & \frac{46}{15} & \frac{35}{8} & \frac{353}{120} & 0\end{array}\right]$

is an EDM which is not spherical with eigenvalues

$$
\left[\begin{array}{c}
15.06857044 \\
-1.002439569 \\
-2.001141510 \\
-3.007884565 \\
-4.010967084 \\
-5.046137716
\end{array}\right]
$$

and for
And by using Theorem 2.6, and Example 2.9 in [15], we have $\rho=\sqrt{\left(-\lambda_{n+1}-\lambda_{n+2}\right)\left(\lambda_{1}+\lambda_{n+1}\right)}=\sqrt{\left(-\lambda_{5}-\lambda_{6}\right)\left(\lambda_{1}+\lambda_{5}\right)}=3 \sqrt{11}$, the spectrum of $D_{1}=\left(\begin{array}{cccc}0 & \frac{5}{2} & 2 & \frac{3}{2} \\ \frac{5}{2} & 0 & \frac{3}{2} & 2 \\ 2 & \frac{3}{2} & 0 & \frac{5}{2} \\ \frac{3}{2} & 2 & \frac{5}{2} & 0\end{array}\right)$, is $\{6,-1,-2,-3\}$, and

$$
D=\left[\begin{array}{cccccc}
0 & \frac{3}{2} & \frac{5}{2} & 2 & \frac{3}{4} \sqrt{22} & \frac{3}{4} \sqrt{22} \\
\frac{3}{2} & 0 & 2 & \frac{5}{2} & \frac{3}{4} \sqrt{22} & \frac{3}{4} \sqrt{22} \\
\frac{5}{2} & 2 & 0 & \frac{3}{2} & \frac{3}{4} \sqrt{22} & \frac{3}{4} \sqrt{22} \\
2 & \frac{5}{2} & \frac{3}{2} & 0 & \frac{3}{4} \sqrt{22} & \frac{3}{4} \sqrt{22} \\
\frac{3}{4} \sqrt{22} & \frac{3}{4} \sqrt{22} & \frac{3}{4} \sqrt{22} & \frac{3}{4} \sqrt{22} & 0 & 4 \\
\frac{3}{4} \sqrt{22} & \frac{3}{4} \sqrt{22} & \frac{3}{4} \sqrt{22} & \frac{3}{4} \sqrt{22} & 4 & 0
\end{array}\right],
$$

is an EDM and same matrix (8.1) has spectrum $\sigma$.

$$
W^{\prime}=\left[\begin{array}{ccccc}
1 & -2 & 3 & 4 & -5 \\
-1 & -2 & 3 & -4 & 5 \\
1 & 2 & 3 & 4 & 5 \\
-1 & 2 & -3 & 4 & -5 \\
1 & 2 & -3 & -4 & -5 \\
-1 & -2 & -3 & -4 & 5
\end{array}\right], \quad D=\left[\begin{array}{cccccc}
0 & \frac{67}{24} & \frac{7}{2} & \frac{5}{3} & \frac{25}{8} & \frac{19}{6} \\
\frac{67}{24} & 0 & \frac{73}{24} & \frac{25}{8} & \frac{13}{6} & \frac{31}{8} \\
\frac{7}{2} & \frac{73}{24} & 0 & \frac{19}{6} & \frac{31}{8} & \frac{13}{6} \\
\frac{5}{3} & \frac{25}{8} & \frac{19}{6} & 0 & \frac{67}{24} & \frac{7}{2} \\
\frac{25}{8} & \frac{13}{6} & \frac{31}{8} & \frac{67}{24} & 0 & \frac{73}{24} \\
\frac{19}{6} & \frac{31}{8} & \frac{13}{6} & \frac{7}{2} & \frac{73}{24} & 0
\end{array}\right]
$$

is a distance matrix with eigenvalues

$$
\left[\begin{array}{c}
-3 \\
-2 \\
-1 \\
15.01897846 \\
-5.014117791 \\
-4.004860665
\end{array}\right]
$$

and $\mathrm{D}$ is similar to the matrix

$$
\left[\begin{array}{cccccc}
-1 & 0 & 0 & 0 & 0 & 0 \\
0 & -2 & 0 & 0 & 0 & 0 \\
0 & 0 & -3 & 0 & 0 & 0 \\
0 & 0 & 0 & -4 & 0 & \frac{1}{8} \sqrt{6} \\
0 & 0 & 0 & 0 & -5 & \frac{-1}{8} \sqrt{6} \sqrt{3} \\
0 & 0 & 0 & \frac{1}{8} \sqrt{6} & \frac{-1}{8} \sqrt{6} \sqrt{3} & 15
\end{array}\right] .
$$

\section{Conclusion}

This is important problem that what is the set of eigenvalues is realized by symmetric nonnegative matrix. The necessary conditions said to us that for instance the set of eigenvalues $\{5,-5,0.6,0.5,0.5,-1.5\}$ that although has nonnegative summation but it is easy to see that for large odd $k$ has negative summation $s_{k}=\Sigma_{i=1}^{6} \lambda_{i}^{k}$ then for this example we have no solution. Therefore, we can say that if for every set of eigenvalues, that has nonnegative sumation and holds in condition (2) of necessary conditions, then the problem is solved.

Acknowledgements The authors wish to thank an anonymous referee, whose sensible suggestions led to a significant rewrite which greatly simplified the overall presentation of the results.

Open Access This article is licensed under a Creative Commons Attribution 4.0 International License, which permits use, sharing, adaptation, distribution and reproduction in any medium or format, as long 
as you give appropriate credit to the original author(s) and the source, provide a link to the Creative Commons licence, and indicate if changes were made. The images or other third party material in this article are included in the article's Creative Commons licence, unless indicated otherwise in a credit line to the material. If material is not included in the article's Creative Commons licence and your intended use is not permitted by statutory regulation or exceeds the permitted use, you will need to obtain permission directly from the copyright holder. To view a copy of this licence, visit http://creativecommons.org/licenses/by/4.0/.

\section{References}

1. Fiedler, M.: Eigenvalues of nonnegative symmetric matrices. Linear Algebra Appl. 9, 119-142 (1974)

2. Smigoc, Helena: The inverse eigenvalue problem for nonnegative matrices. Linear Algebra Appl. 393, 365-374 (2004)

3. Reams, R.: An inequlity for nonnegative matrices and the inverse eigenvalue problem. Linear Multilinear Algebra 41, 367-375 (1996)

4. Laffey, T.J., Meehan, E.: A characterization of trace zero nonnegative $5 \times 5$ matrices. Linear Algebra Appl. 302-303, 295-302 (1999)

5. Radwan, N.: An inverse eigenvalue problem for symmetric and normal matrices. Linear Algebra Appl. 248, 101-109 (1996)

6. Smigoc, Helena: Construction of nonnegative matrices and the inverse eigenvalue problem. Linear Multilinear Algebra 53(2), 85-96 (2005)

7. Torre-Mayo, J., Abril-Raymundo, M.R., Alarcia-Estevez, E., Marijuan, C., Pisonero, M.: The nonnegative inverse eigenvalue problem from the coefficients of the characteristic polynomial. EBL Digraphs Linear Algebra Appl. 426, 729-773 (2007)

8. Meehan, E.: Some results on matrix spectra. Ph.D. thesis, University College Dublin (1998)
9. Rojo, Oscar, Soto, Ricardo L.: Existence and construction of nonnegative matrices with complex spectrum. Linear Algebra Appl 368, 53-69 (2003)

10. Soto, R.L., Julio, A.I.: A note on the symmetric nonnegative inverse eigenvalue problem. Int. Math. Forum 6(50), 2447-2460 (2011)

11. Soto, R.L., Rojo, O., Borobia, A., Moro, J.: Symmetric nonnegative realization of spectra. Electron. J. Linear Algebra 16, 1-18 (2007)

12. Laffey, T.J., Smigoc, H.: Construction of nonnegative symmetric matrices with given spectrum. Linear Algebra Appl 421, 97-109 (2007)

13. Hayden, T.L., Reams, R., Wells, J.: Methods for constructing distance matrices and the inverse eigenvalues problem. Linear Algebra Appl 295, 97-112 (1999)

14. Jaklič, G., Modic, J.: A note on "Methods for constructing distance matrices and the inverse eigenvalue problem". Linear Algebra Appl 437, 2781-2792 (2012)

15. Nazari, A.M., Mahdinasab, F.: Inverse eigenvalue problem of distance matrix via orthogonal matrix. Linear Algebra Appl 450, 202-216 (2014)

16. Lowey, R., London, D.: A note on an inverse problem for nonnegative matrices. Linear Multilinear Algebra 6, 83-90 (1978)

17. Johnson, C.R.: Row stochastic matrices similar to doubly stochastic matrices. Linear Multilinear Algebra 10(2), 113-130 (1981)

18. Nazari, A.M., Sherafat, F.: On the inverse eigenvalue problem for nonnegative matrices of order two to five. Linear Algebra Appl 436, 1771-1790 (2012)

Publisher's Note Springer Nature remains neutral with regard to jurisdictional claims in published maps and institutional affiliations. 